\title{
Supersonic Similitude for Oscillating Nonplanar Wedge
}

\author{
Asha Crasta ${ }^{1}$ and S. A. Khan ${ }^{2}$ \\ Research Scholar, Department of Mathematics, Jain University, Bangalore, Karnataka, India, \\ Principal, Department of Mechanical Engineering, Bearys Institute of technology, Mangalore, Karnataka, \\ India,
}

\begin{abstract}
A similitude has been obtained for a pitching oscillating Nonplanar wedge with attached bow shock at high angle of attack in supersonic flow. A strip theory in which flow at a span wise location is two dimensional and independent of each other is being used. This combines with the similitude to lead to a onedimensional piston theory. Closed form of simple relations is obtained for stiffness and damping derivatives in pitch. The present theory is valid only when the shock wave is attached with the nose of the wedge. From the theory developed some of the results are obtained for wide range of Mach number and angle of attack with remarkable computational ease. From the results it is found that when convexity is introduced in the non-planar wedge, this results in shifting of the center of pressure towards the leading edge, and the stiffness as well as the damping derivative decreases with the increase in the Mach number for all the values of the semi vertex angles.
\end{abstract}

Keywords: Supersonic Flow, Non Planar wedge, Piston Theory, pitch

\section{Introduction:}

High incidence hypersonic similitude ofSychev's [1] is applicable to a wing provided it has an extremely small span in addition to small thickness. The unsteady infinite span case has been analyzed, but mostly for small flow deflections. The piston theory of Light hill [2] neglects the effects of secondary wave reflection. Appleton [3] and McIntosh [4] have included these effects. Hui's [5] theory is valid for wedges of arbitrary thickness oscillating with small amplitude provided the bow shock remains attached. Erricsson's [6] theory covers viscous and elastic effects for airfoils with large flow deflection. Orlik-Ruckemann [7] has included viscous effect and Mandl[8] has addressed small surface curvature effect for oscillating thin wedges. Ghosh's [9] similitude and piston theory for the infinite span case with large flow deflection is valid for airfoils with planar or non-planar surfaces whereas Hui's theory[10] is for plane wedges. Ghosh's piston theory has been applied to non-planar cases, both steady and unsteady. The effect of viscosity and secondary wave reflection has not been included.Crasta and Khan have studied the hypersonic and supersonic similitude for planar wedge([17],[12]), for Delta wing ([11],[13])and for Delta wing with curved leading edges([14],[16]). Crasta and Khan have further extended the similitude tostudy the stability derivatives for Newtonian limit for planar wedge, delta wing[18] and delta wing with curved leading edges[15]. In the present work the similitude of Supersonic planar wedge has been extended for supersonic flows past a non-planar wedge.

\section{Analysis:}

\section{STEADY WEDGE:}

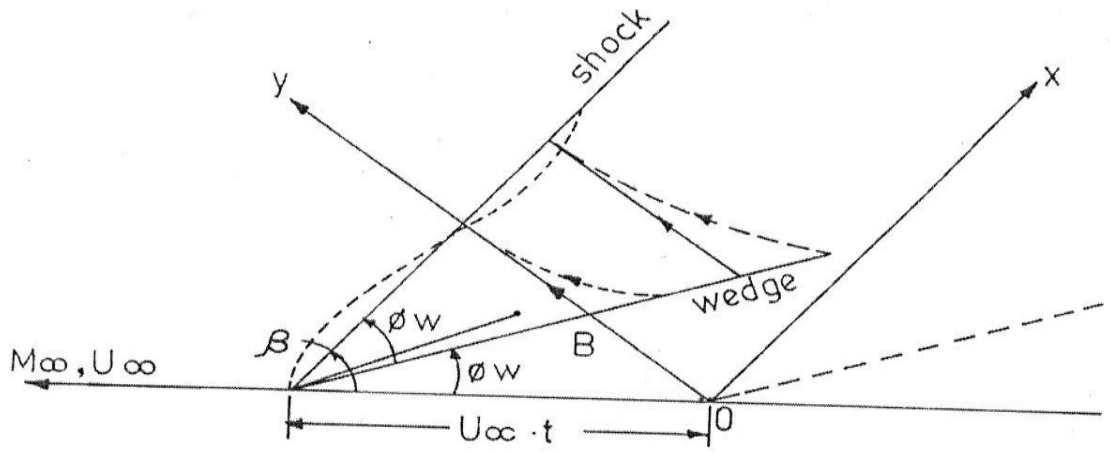

Fig. A shows the wedge at time t.

Fig. A shows that upper half of a steady wedge with attached bow shock in rectilinear flight from right to left in stationary air, at time t. Dimensional analysis indicates that the flow is conical in nature, i.e., at a given instant 
$\frac{\partial}{\partial r}=0$ where $\mathrm{r}$ is the distance along a ray from the apex. Hence the bow shock must coincide with array. The space-fixed co-ordinate system $(\mathrm{x}, \mathrm{y})$ is so chosen that the $\mathrm{x}$-axis coincides with the bow shock at time $\mathrm{t}=0$. Conicality of the flow implies that the instantaneous streamlines have the same slope where they intersect a ray from the apex. Since the shock sets the fluid particles in motion normal to itself, the instantaneous streamlines intersect the shock at right angles. The dashed lines in fig. 1 are probable streamline shapes. We tentatively assume that the streamlines are straight shown by firm lines in Fig.1, if this leads to $\frac{\partial}{\partial r}=0$ then it is a solution. Consider the plane flow on stream surface $\mathrm{x}=0$. At time $\mathrm{t}$ the shock location on $\mathrm{x}=0$ is $y_{s}=U_{\infty} t \sin \beta(1)$

And the body location can be shown to be $Y_{b}=O B=U_{\infty} t \sin \theta_{w} / \cos \theta_{w}$.

Since the flow in plane $\mathrm{x}=0$ is independent of the flow in a neighboring parallel plane, it can be taken as a piston driven fluid motion where the piston Mach number $M_{p}=M_{\infty} \sin \theta_{w} / \cos \left(\beta-\theta_{w}\right)$ and the shock Mach number $M_{s}=M_{\infty} \sin \beta$. Since $M_{p}$ is independent of t, pressure remains constant in this 1D space. Therefore, $\partial p / \partial y=0$. Since the streamlines are straight there is no centrifugal force; thus $\partial p / \partial x=0$. Hence, $\partial p / \partial r=0$. Thus the wedge flow is exactly equivalent to $1 \mathrm{D}$ piston motion normal to the shock. It can be shown that the relation between $M_{S} \& M_{P}$ yields the well known oblique shock relation giving the shock relation giving the shock angle in terms of $\theta_{w}$.

\section{QUASI-WEDGE OR OSCILLATING WEDGE:}

Fig. A shows the probable shape of the bow shock in dotted when the wedge is either oscillating or replaced by a quasi-wedge. The slope of the curved shock with $\mathrm{x}$-axis remains small, say of order $\phi$. Let the Mach number behind the shock, in body-fixed coordinate, be $M_{2}$. The characteristics make an angle $\delta=\left(\sin ^{-1} 1 / M_{2}-\phi_{W}\right)$ with the $\mathrm{x}$-axis. It can be shown that this angle remains small for fairly large values of $\theta_{W}$ even for moderate Mach numbers. For example for $M_{\infty}=2, \theta_{W}=15^{\circ}$, we have $\delta=13^{\circ}$.

Again for $\mathbf{M}_{\infty}=\mathbf{3}, \theta_{W}=20^{\circ}$ we get $\delta=12.5^{\circ}$. we stipulate (see Fig.A) that $\delta \leq 0.3, \quad$ and then $\phi \& \delta$ are of same order. Since the gradient is normal to the characteristics we have,

$\frac{\partial}{\partial x}=0\left(\phi \cdot \frac{\partial}{\partial y}\right)(3)$

Also the net perturbation introduced by the shock and Mach waves will chiefly be in the y-direction. Thus $\mathrm{u}=0(\phi \cdot v)(4)$

Where, $\mathrm{u}$ and $\mathrm{v}$ are velocity components in $\mathrm{x} \& \mathrm{y}$ directions. Equations (3) \& (4) suggest transformations $u^{1}=\phi^{-1} . u \& x^{1}=\phi, x$.

We apply these transformations to the equation of continuity to get $\frac{\partial \rho}{\partial t}+\frac{\partial(\rho v)}{\partial y}=-\phi^{2} \frac{\partial\left(\rho u^{1}\right)}{\partial x^{1}}$.

Similarly, applying these transformations to the rest of the equations of motion and boundary conditions and neglecting terms of $0\left(\phi^{2}\right)$, we get equivalence with a $1 \mathrm{D}$ piston motion in y-direction and unified supersonic/hypersonic similitude.

\section{PISTON THEORY:}

A thin strip of the wing, parallel to the centerline, can be considered independent of the $\mathrm{z}$ dimension when the velocity component along the $\mathrm{z}$ direction is small. This has been discussed by Ghosh's [9]. The strip theory combined with Ghosh's large incidence similitude leads to the "piston analogy" and pressure P on the surface can be directly related to equivalent piston mach no. $\mathrm{M}_{\mathrm{p}}$. In this case both $\mathrm{Mp}$ and flow deflections are permitted to be large. Hence light hill piston theory[2] or miles strong shock piston theory cannot be used but Ghosh's piston theory will be applicable. 


$$
\frac{P}{P_{\infty}}=1+A M_{P}{ }^{2}+A M_{P}\left(B+M_{P}{ }^{2}\right)^{\frac{1}{2}} \quad \text {, Where } P_{\infty} \text { is free stream pressure }
$$

Since strips at different span wise location are assumed independent of each other, the strip can be considered as a flat plate at an angle of attack. The angle of incidence is same as that of wing. Angle $\phi$ is the angle between the shock and the strip. A piston theory which has been used in equation(7) has been extended to supersonic flow. The expression is given below.

$$
\frac{p}{p_{\infty}}=1+A\left(\frac{M_{p}}{\cos \phi}\right)^{2}+A\left(\frac{M_{p}}{\cos \phi}\right)\left(B+\left(\frac{M_{p}}{\cos \phi}\right)^{2}\right)^{\frac{1}{2}}
$$

Where $p_{\infty}$ is free stream pressure, $A=\frac{(\gamma+1)}{4}, B=\left(4 /(\gamma+1)^{2}, \gamma\right.$ is the specific heat ratio and $M_{p}=$ the local piston Mach number normal to the wedge surface.

\section{Pitching moment derivatives}

Let the mean incidence be $\alpha_{0}$ for the wing oscillating in pitch with small frequency and amplitude about an axis $\mathrm{X}_{0}$. The piston velocity and hence pressure on the windward surface remains constant on a span wise strip of length $2 \mathrm{~L}$ at $\mathrm{x}$, the pressure on the lee surface is assumed zero. Therefore, the nose up moment is

$$
m=-\int_{0}^{L} p . .\left(x-x_{0}\right) d x
$$

A non planar wedge is obtained by superimposing parabolic arcs on the two sides of the plane wedge.

Therefore The Stiffness and damping derivatives are respectively

$$
\begin{aligned}
& -C_{m_{\alpha}}=\frac{2}{\frac{1}{2} \rho_{\infty} U \infty^{2} L^{2} \cos \phi}\left(-\frac{\partial m}{\partial \alpha}\right)_{\alpha=\alpha_{0}, q=0} \\
& -C_{m_{q}}=\frac{2}{\frac{1}{2} \rho_{\infty} U \infty^{2} L^{3} \cos \phi}\left(-\frac{\partial m}{\partial q}\right)_{\alpha=\alpha_{0}, q=0}
\end{aligned}
$$

Where $\rho_{\infty}, \mathrm{a}_{\infty}$ are density and velocity of sound in the free stream? Combining (7) through (10), differentiation under the integral sign is performed. Therefore

$$
-C_{m_{\alpha_{0}}}=\frac{p_{\infty} \cdot A}{\frac{1}{2} \rho_{\infty} U_{\infty}{ }^{2} L^{2} \operatorname{Cos}^{2} \alpha_{0} \cos \phi_{0}^{L}} \int_{0}^{L}\left(x-x_{0}\right) \cdot \frac{\partial p}{\partial \alpha} \cdot d x
$$

And

$$
-C_{m_{q}}=\frac{p_{\infty} \cdot A}{\frac{1}{2} \rho_{\infty} U_{\infty} L^{3} \operatorname{Cos}^{3} \alpha_{0} \cos \phi} \int_{0}^{L}\left(x-x_{0}\right) \cdot \frac{\partial p}{\partial q} \cdot d x
$$

On Simplifying we get,

The Stiffness and damping derivative are given by 


$$
\begin{gathered}
-C_{m_{\alpha}}=\frac{\gamma+1}{2 M_{\infty}^{2} \cos ^{2} \alpha_{0} \cos \phi}\left(I_{1}+I_{2}+I_{3}\right) \\
-C_{m_{q}}=\frac{\gamma+1}{2 M_{\infty} \cos ^{3} \alpha_{0} \cos \phi}\left(J_{1}+J_{2}+J_{3}\right)
\end{gathered}
$$

Where

$$
I_{1}=\frac{M_{\infty}^{2}}{\cos \phi}\left[H \sin 2 \alpha_{0}-\frac{2 \lambda \cos 2 \alpha_{0}}{3}\right]
$$$$
I_{2}=\left[\left\{-M_{\infty} \frac{(z)^{\frac{3}{2}}}{3 b}\right\}\left\{H \cos \alpha_{0}-\left(H \lambda \sin \alpha_{0}+\cos \alpha_{0}\right)\left(\frac{5 a-3 z}{5 b}\right)+\lambda \sin \alpha_{0}\left(\frac{15 z^{2}-42 a z+35 a^{2}}{3 b^{2}}\right)\right\}\right]_{z=a+b}^{z=a-b}
$$$$
I_{3}=\left[\left\{\frac{M_{\infty} z^{\frac{1}{2}}}{2 \lambda \cos \alpha_{0}}\right\}\left\{-H \sin \alpha_{0} \cos \alpha_{0}+\left(\begin{array}{l}
\left.\left(\sin \alpha_{0} \cos \alpha_{0}-\lambda H\left(\cos ^{2} \alpha_{0}+\cos 2 \alpha_{0}\right)\right)\left(\frac{z-3 a}{3 b}\right)\right) \\
+\lambda\left(\cos ^{2} \alpha_{0}+\cos 2 \alpha_{0}\right)\left(\frac{3 z^{2}-10 a z+15 a^{2}}{15 b^{2}}\right)
\end{array}\right)\right\}\right]_{z=a+b}^{z=a-b}
$$

$$
J_{1}=M_{\infty}\left[H^{2} \sin \alpha_{0}+\left(\frac{\sin \alpha_{0}-2 H \lambda \cos \alpha_{0}}{3}\right)\right]
$$

$$
J_{2}=\frac{-2 z^{\frac{3}{2}}}{3 b}\left[\frac{H^{2}}{4}+H\left(\frac{5 a-3 z}{10 b}\right)+\frac{15 z^{2}-42 a z+35 a^{2}}{140 b^{2}}\right]_{z=a+b}^{z=a-b}
$$

$$
J_{3}=\left[\left\{\frac{-z}{\lambda \cos \alpha_{0}}\right\}\left\{\begin{array}{l}
\left(\frac{H^{2} \sin \alpha_{0}}{4}\right)-H\left(\sin \alpha_{0}-\lambda H \cos \alpha_{0}\right)\left(\frac{z-3 a}{6 b}\right) \\
+\left(\sin \alpha_{0}-4 \lambda H \cos \alpha_{0}\right)\left(\frac{3 z^{2}-10 a z+15 a^{2}}{60 b^{2}}\right) \\
+\lambda \cos \alpha_{0}\left(\frac{5 z^{3}-21 z^{2} a+35 z a^{2}-35 a^{3}}{70 b^{3}}\right)
\end{array}\right\}\right]_{z=a+b}^{z=a-b}
$$

Where 


$$
\begin{aligned}
H & =1-2 h \cos ^{2} \alpha_{O} \\
\mathbf{a} & =\left(\frac{4}{\gamma+1}\right)^{2}+\frac{M^{2} \infty \sin ^{2} \alpha_{O}}{\cos \phi}, \\
\mathrm{b} & =\frac{\lambda \mathrm{M}^{2} \infty \sin 2 \alpha_{O}}{\cos \phi}
\end{aligned}
$$

Some of the results have been obtained for various angle of incidences and Mach Numbers.

\section{RESULTS AND DISCUSSIONS:}

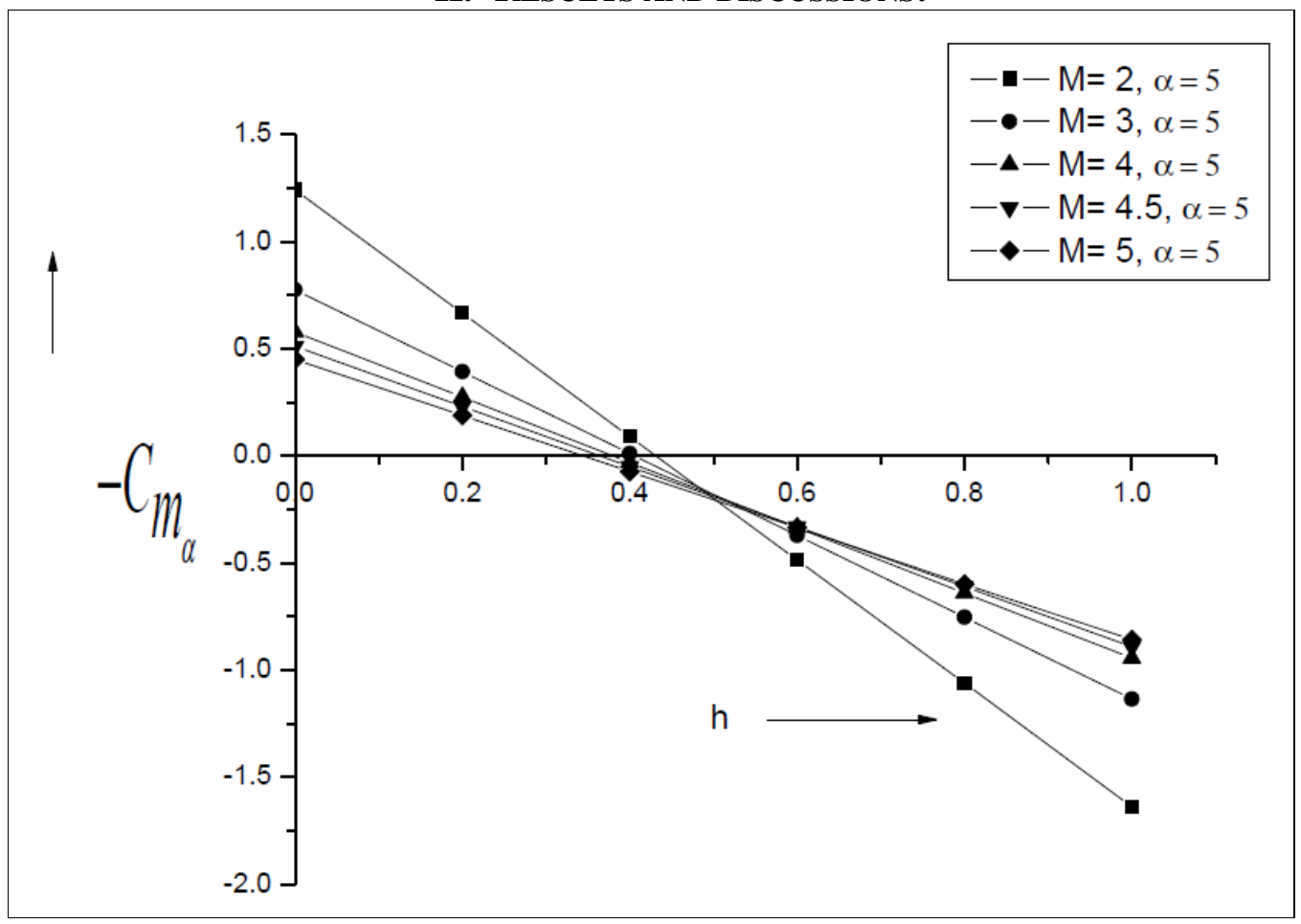

Fig. 1: Variation of stiffness derivative with pivot position

Fig.1 Shows the variation of Stiffness derivative with respect to pivot position for different Mach number while the semi vertex angle is kept constant $\left(\alpha_{0}=5\right)$. From the figure it is seen that with the increase in Mach number from $M=2$ to 5 there is continuous decrease in the stiffness derivative. This is also observed that the location of center of pressure is moving towards the leading edge which; means that from the static stability point of view for a given semi vertex angle increase in Mach number results in the decrease of stiffness derivative as well as the center of pressure shifts towards the leading edge. From the figureit is seen that there is decrease in stiffness derivative which is $58 \%$ between $\mathrm{M}=2$ to 3 , around $36 \%$ for the Mach number range $\mathrm{M}=3$ to 4 , around $10 \%$ for Mach number range $M=4$ to 4.5 , and around $25 \%$ for the Mach number range $M=4.5$ to 5 respectively. The Variation of center of pressure is $35 \%$ to $45 \%$ for Mach Number 2,3,4,4.5, and 5 respectively from the Nose position, since the semi vertex angle is only five degrees. The wedge falls under the category of slender body with a very high length to diameter ratio. In this particular case the shock wave angle are small compared to that for semi vertex angle in the range of 20 degrees and above. For very high value of semi vertex angle the shock wave may not be attached with body. Instead of oblique shock there will be a bow shock at the nose of the planar wedge. Due to the presence of oblique shock the flow behind the shock will be subsonic and flow separation will take place. The present theory is valid only when the shock wave is attached with body that is at the nose of the wedge. Further the present theory is quasi steady where as the theory of Hui and Lui is fully unsteady which is more accurate. This difference is observed only in the case of damping derivatives. For the stiffness derivatives the results are identical. 


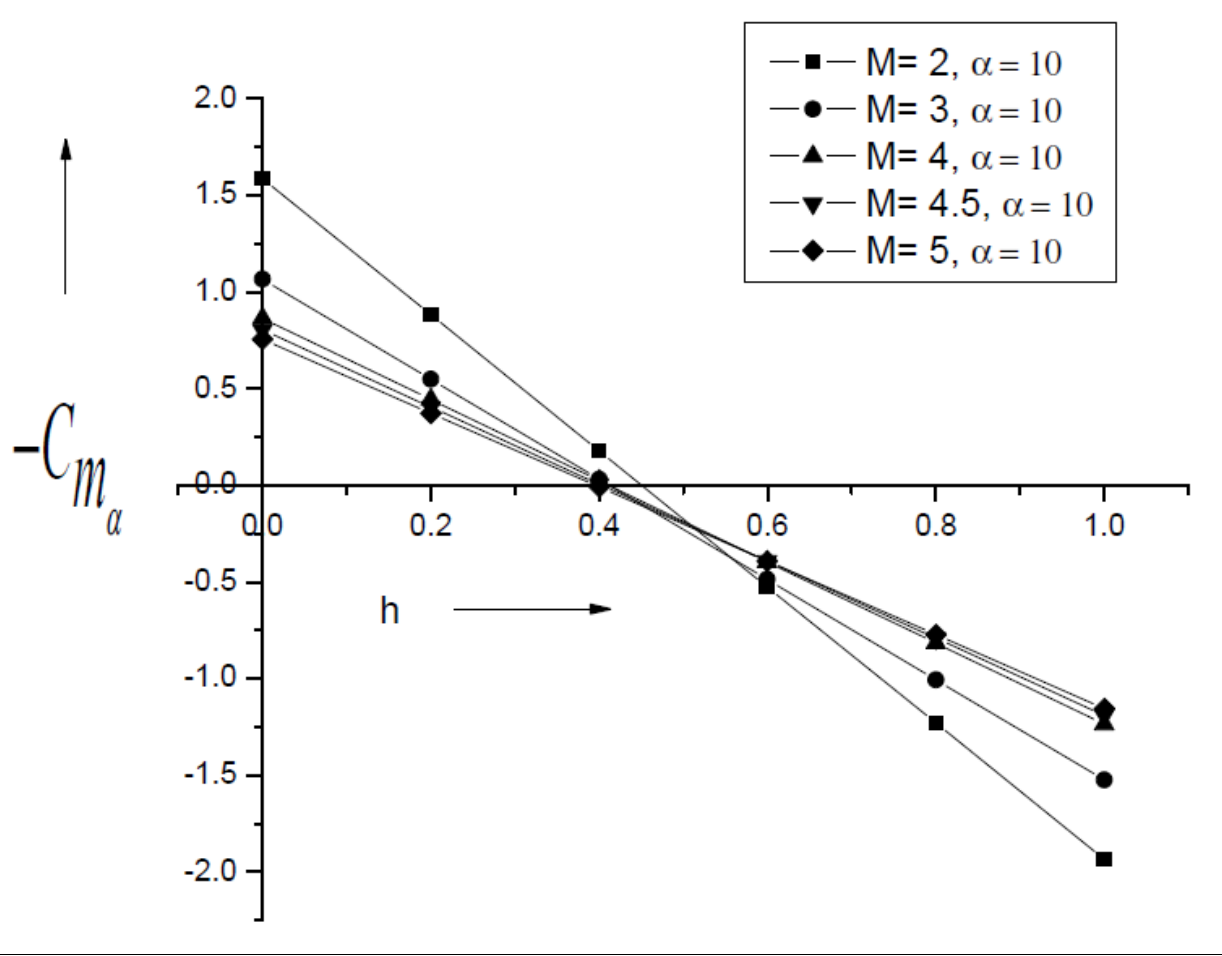

Fig. 2: Variation of stiffness derivative with pivot position

Fig. 2 shows the results for stiffness derivatives for the same range of Mach numbers $M=2,3,4,4.5$, and 5 for semi vertex angle ten degrees discussed. From the figure 2 it is seen that there is $31 \%$ decrease in the stiffness derivative for the Mach number range $M=2$ to 3 , it is around $19 \%$ for the Mach number range $M=3$ to 4 , it is around $8 \%$ each for the Mach number range $M=4$ to $4.5 \& 4.5$ to 5 . It is also seen that the center of pressure is varying from $40 \%$ to $45 \%$ from the leading edge. There is overlapping of values and the reason for this trend may be due to the low Mach number range of the present study which; does not result in substantial change in the surface pressure flow field of the wedge.

Results for stiffness derivatives for semi vertex angle 15 degrees are shown in Fig 3. It is found that the reduction in the stiffness derivative is around $27 \%$ for the Mach number range $\mathrm{M}=2$ to 3 , around $15 \%, 10 \%$, and $6 \%$ for the Mach number range $\mathrm{M}=3$ to 4, 4 to 4.5 , and 4.5 to 5, respectively. There is progressive decrease in the values of Stability derivatives. Further there is a linear shift in the center of pressure towards the trailing edge of the wedge due to the area shift towards the trailing edge.

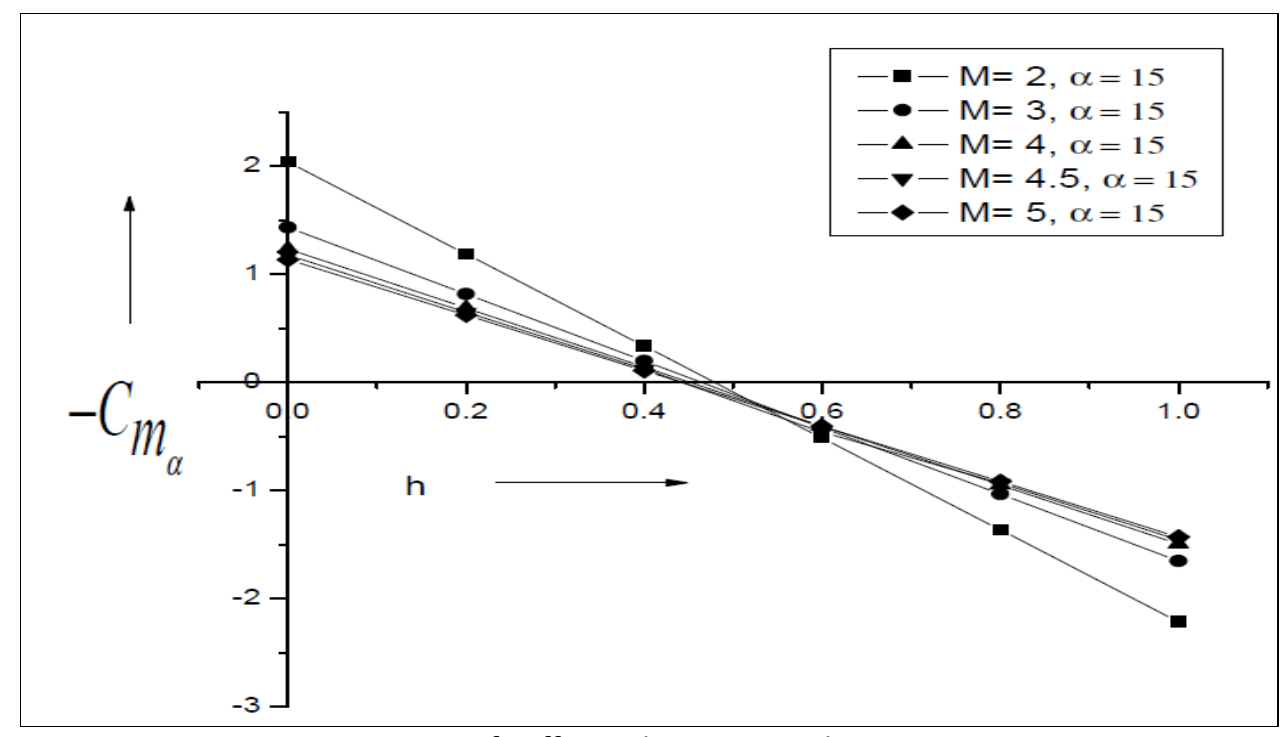

Fig 3: variation of stiffness derivative with pivot position 


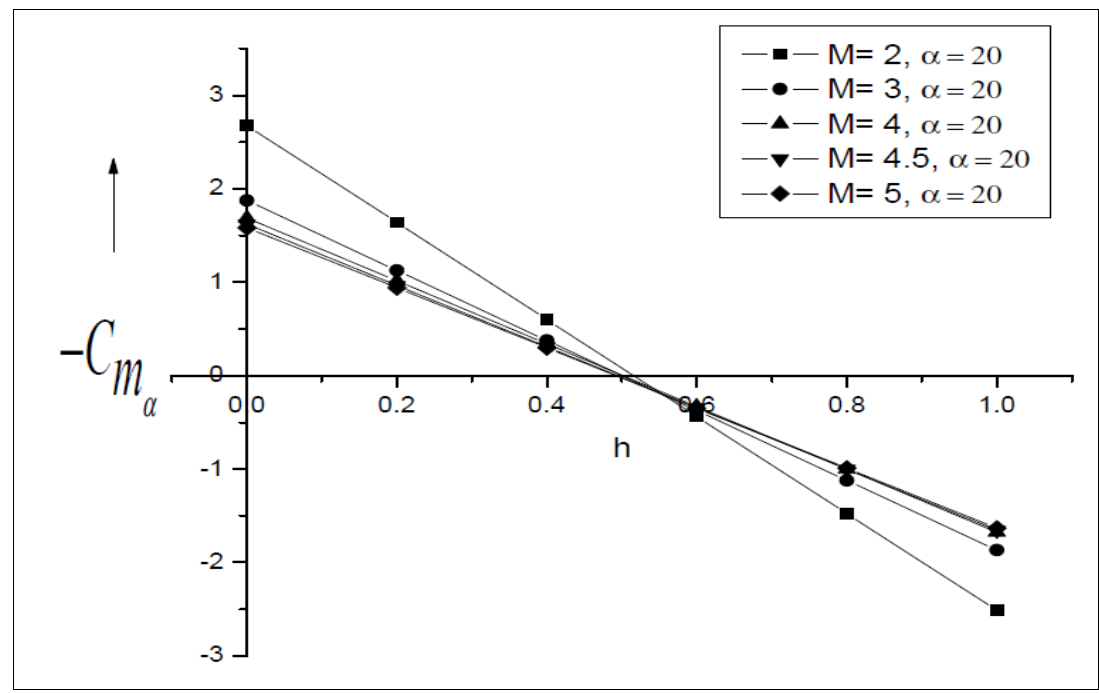

Fig. 4: Variation of stiffness derivative with pivot position

Fig. 4 shows the variation of stiffness derivative with pivot position forsemivertex angle 20 degrees. The Stiffness derivative at nose is the highest at $\mathrm{M}=2$ and decreases with increase in the Mach number.It is found that there is decrease in stiffness derivative around $27 \%, 8 \%, 5 \%$, and $2 \%$ for the Mach number ranges $\mathrm{M}=2$ to $3, \mathrm{M}=3$ to $4, \mathrm{M}=4$ to 4.5 , and $\mathrm{M}=4.5$ to 5 , respectively. The center of pressure of the non-planar wedge lies in the range $50 \%$ to $55 \%$ from the leading edge. This implies that the stability of a non planar wedge decreases as Mach number increases. All the curves almost overlap with each other. This behavior may be due to the Mach number chosen for the particular case. The value of the surface pressure and the Mach number range of the present study lie in supersonic zone. The present theory is an extension of Hypersonic Flow theory. The amount of error involved will be on the higher side. It is seen from the above figures that the center of pressure for all the Mach number remains at $50 \%$ for stiffness.

Fig. 5 shows the variation of damping derivative with respect to the pivot position for different Mach numbers. It is noted that damping derivative at nose is highest for $\mathrm{M}=2$ and decreases with increase in Mach number. Damping derivative is lowest at centre of pressure. It is found that the damping is decreased by around $36 \%, 33$ $\%, 12 \%$, and $10 \%$ for the Mach numbers in the range 2 to 3, 3 to 4, 4 to 4.5 , and 4.5 to 5, respectively, and the minima which happens to the aerodynamic center remains around at $40 \%$ from the leading edge of the wedge for all the Mach numbers of the present study. From these results it is seen that the values of the damping derivatives in the leading edge locations assumes low values for all the Mach numbers and these values remain in the range from 0.4 to 1 ,however, once the damping derivatives have the minimum values; then in the downstream the magnitude of the damping assumes very high values as compared to those obtained for the leading edge area. The values in the downstream are from 0.2 to 1.3 . The drastic change in the magnitude of the damping derivatives may be due the pressure distribution pattern on the non-planar wedge surface for the present range of the Mach numbers and the angle of attack. This change in the magnitude of the damping derivatives assumes importance from the dynamic stability point of view.

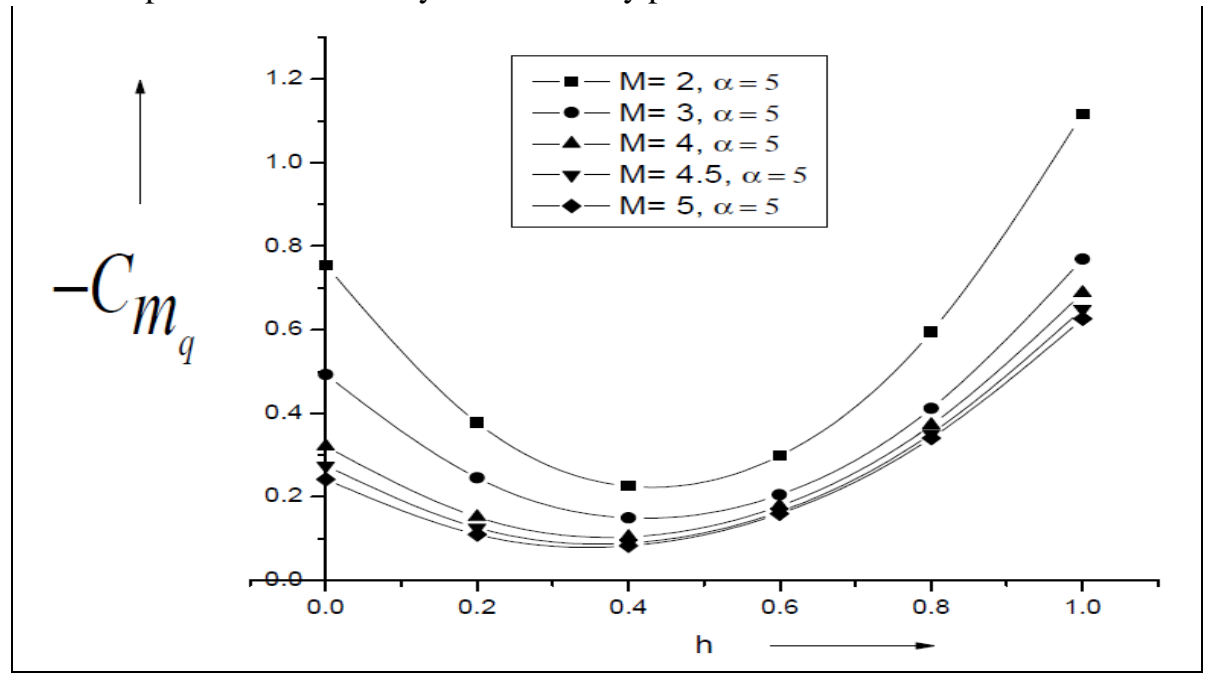

Fig. 5: Variation of damping derivative with pivot position 
Fig. 6 presents the results for damping derivatives in pitch for Mach numbers from $M=2$ to 5 and at angle of attack ten degrees. From the results it is found that there is continuous decrease in the magnitude of the damping derivatives and they are $32 \%$ for Mach number range [2 to 3], $23 \%$ for Mach number range [3 to 4], $10 \%$ for Mach number range [4 to 4.5], and $7 \%$ for the Mach number range [4.5 to 5]. It is also seen that at the trailing edge of the wedge the values have increased appreciably.

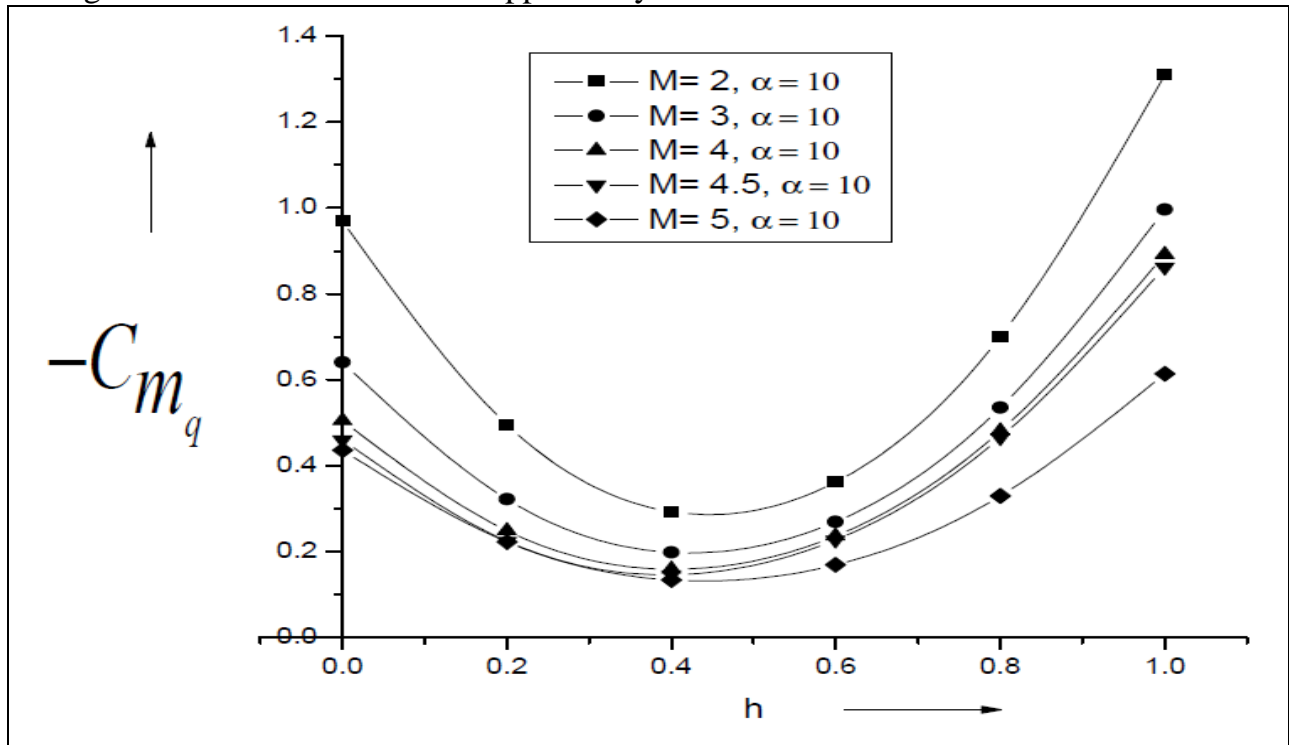

Fig. 6 : Variation of damping derivative with pivot position

Fig. 7 presents the results for damping derivatives in pitch for Mach numbers from $M=2$ to 5 and at angle of attack fifteen degrees. From the results it is found that there is continuous decrease in the magnitude of the damping derivatives and they are $31 \%$ for Mach number range [2 to 3], $15 \%$ for Mach number range [3 to 4], $10 \%$ for Mach number range [ 4 to 4.5], and $7 \%$ for the Mach number range [4.5 to 5]. It is also seen that at the trailing edge of the wedge the values have increased appreciably as compared to the lower semi vertex angle or angle of attack when we consider half of the wedge from the axi-symmetry point of view. As discussed earlier for this case also, the minima of the damping derivative takes place at non-dimensional pivot position of $\mathrm{h}=0.4$.

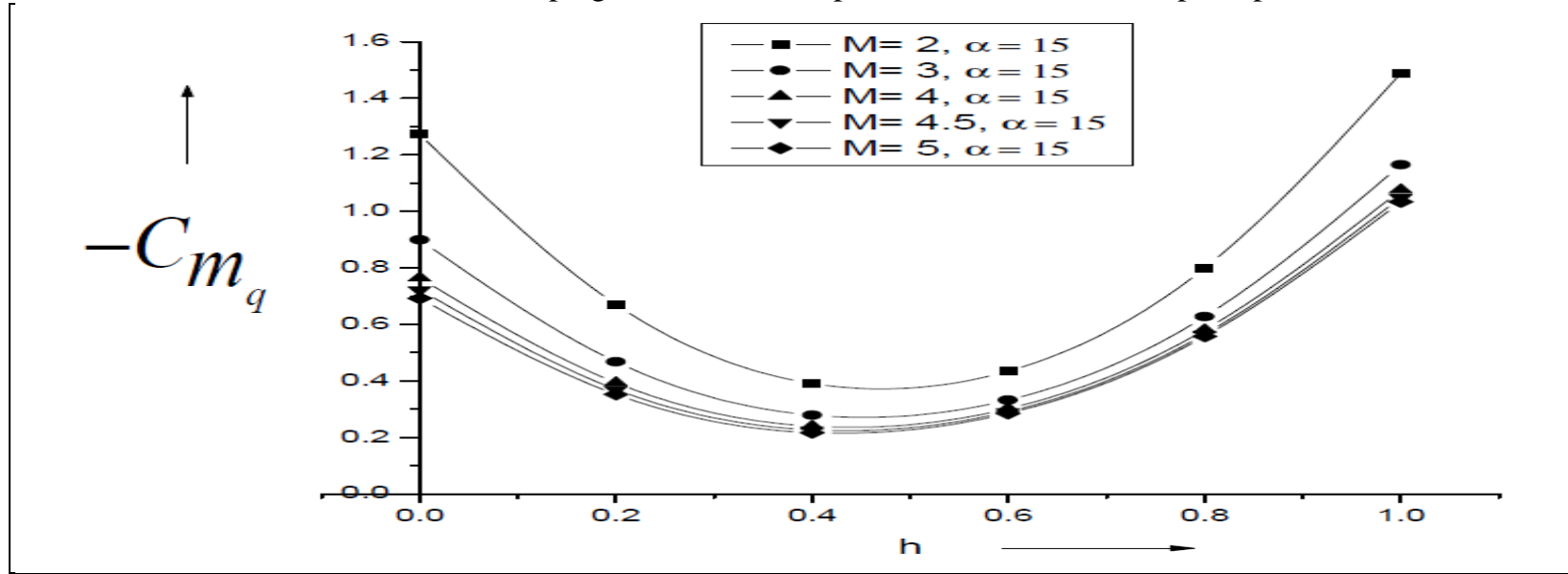

Fig. 7: Variation of damping derivative with pivot position

Fig. 8 presents the results for damping derivatives in pitch for Mach numbers from $M=2$ to 5 and at angle of attack twenty degrees. From the results it is found that there is continuous decrease in the magnitude of the damping derivatives and they are $29 \%$ for Mach number range [2 to 3], $12 \%$ for Mach number range [3 to 4], 6 $\%$ for Mach number range [4 to 4.5], and $4 \%$ for the Mach number range [4.5 to 5]. It is also seen that at the trailing edge of the wedge the values have increased to a great extent as compared to the lower semi vertex angle or angle of attack when we consider half of the wedge from the axi-symmetry point of view. The minima of the damping derivative takes place at non-dimensional pivot position of $h=0.5$, for this case there is a shift in the location of center of pressure towards the trailing edge which is definitely a positive sign. As regards the dynamic stability is concerned this case will have an edge over all the previous cases namely for semi vertex angles five degrees, ten degrees, and fifteen degrees and will result in a better stability. 


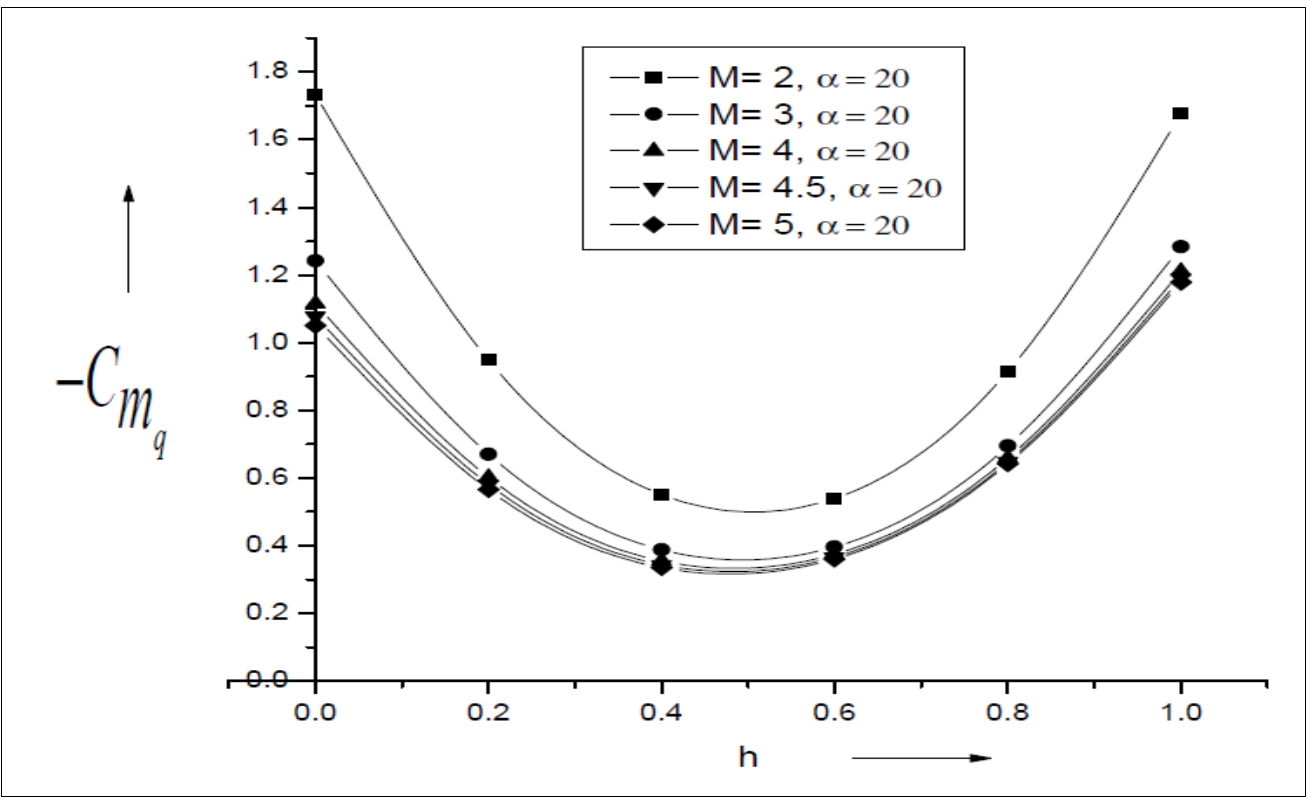

Fig. 8: Variation of damping derivative with pivot position

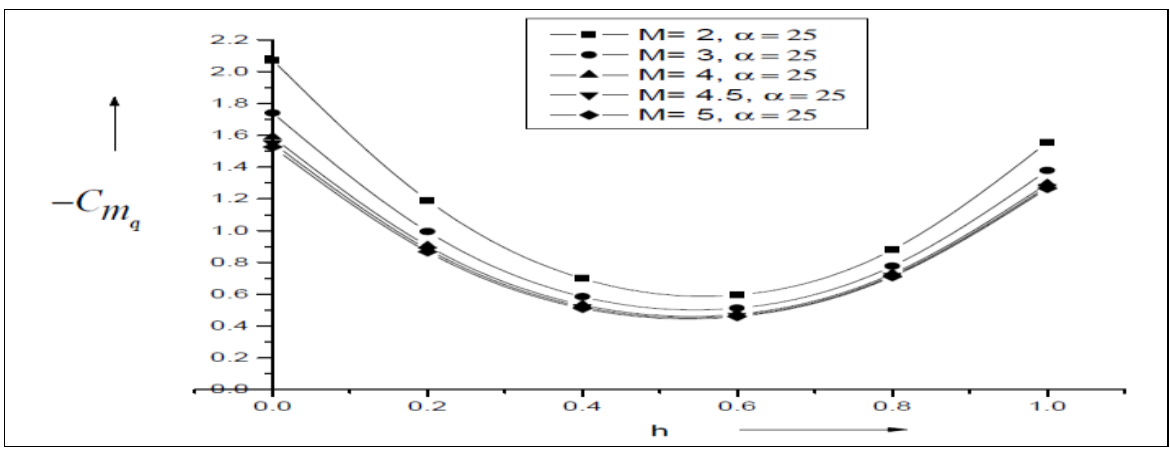

Fig. 9: Variation of damping derivative with pivot position

Fig. 9 presents the results for damping derivatives in pitch for Mach numbers from $M=2$ to 5 and at angle of attack twenty five degrees. From the results it is found that for this particular case the damping derivative has assumed very high value as compared to the previous cases, however, the percentage decrease has come down as compared to the earlier cases for all the Mach number especially in the range [4 to 5]. It is also observed that there is continuous decrease in the magnitude of the damping derivatives and they are $17 \%$ for Mach number range [2 to 3], $10 \%$ for Mach number range [3 to 4], $5 \%$ for Mach number range [4 to 4.5 ], and $4 \%$ for the Mach number range [4.5 to 5]. It is also seen that at the leading edge of the wedge the values have increased to a great extent as compared to the lower semi vertex angle or angle of attack when we consider half of the wedge from the axi-symmetry point of view. The minima of the damping derivative takes place at non-dimensional pivot position of $\mathrm{h}=0.55$, for this case there is a shift in the location of center of pressure towards the trailing edge which is definitely it is to our advantage. As regards the dynamic stability is concerned this case will have better stability as compared to all the previous cases namely for semi vertex angles five degrees, ten degrees, fifteen degrees, and twenty degrees.

\section{Conclusion:}

The Present theory demonstrates the theory's wide application range in incidence and Mach number for Non Planar Surfaces. It is free from the restrictions of Lighthill's theory and Miles theory. The present theory shows that the effect of convexity in non-planar wedges is to decrease stiffness as well as the damping derivatives, and shift damping minima towards the leading edge. This shift in the center of pressure for stiffness as well as the damping derivatives is due the change in the geometry of the wedge, and it is expected that if the non-planar wedge becomes concave instead of convex the trend will get reversed; the center of pressure will shift towards the trailing edge instead of leading edge. In this situation the damping derivative will be attain the maximum value at the leading edge instead of trailing edge. In the present theory the effect of Lee surface is neglected and effect of viscosity and secondary wave reflections have been neglected. 


\section{References:}

[1]. Sychev, V. V, Three Dimensional Hypersonic Gas Flow Past Slender Bodies at High Angles of Attack, Journal of Applied Mathematics and Mechanics, Vol. 24, Aug. 1960,pp.296-306.

[2]. LightHill, M.J., Oscillating Aerofoil at High Mach Numbers, Journal of Aeronautical Sciences, Vol.20, June 1953,pp.402-406.

[3]. Appleton,J.P. , Aerodynamic Pitching Derivatives of a wedge in Hypersonic Flow, AIAA Journal, Vol. 2,Nov.1964, pp.20342036.

[4]. McInthosh, S.C.,Jr., Studies in Unsteady Hypersonic Flow Theory, Ph.D. Dissertation Stanford Univ., calif ,Aug. 1965.

[5]. Hui, W.H., Stability of Oscillating Wedges and Caret Wings in Hypersonic and Supersonic Flows,AIAA Journal, Vol. 7, Aug. 1969, pp. 1524-1530.

[6]. Ericsson,L . E., Viscous and Elastic Pertubation Effects on Hypersonic Unsteady Airfoil Aerodynamics, AIAA Journal, Vol.15 Oct. 1977, pp. 1481-1490.

[7]. Orlik-Ruckemann, K. J.,Stability Derivatives of Sharp Wedges in Viscous Hypersonic Flow, AIAA Journal, Vol. 4June 1966, pp. 1001-1007.

[8]. Mandl ,P., Effect of Small Surface Curvature on Unsteady Hypersonic Flow over an Oscillating Thin Wedge, C.A.S.I. Transactions ,Vol.4, No. 1, March 1971, pp. 47-57.

[9]. Ghosh, Kunal. Hypersonic large deflection similitude for oscillating delta wings, Aeronautical Journal, Oct. 1984, pp. 357-361.

[10]. Lui, D. D. and Hui W. H., Oscillating delta wings with attached shock waves, AIAA Journal , June 1977, 15, 6,804-812.

[11]. AshaCrasta and Khan S. A, Oscillating Supersonic delta wing with Straight Leading Edges, International Journal of Computational Engineering Research, Vol. 2, Issue 5, September 2012, pp.1226-1233,ISSN:2250-3005.

[12]. Asha Crasta and Khan S. A., High Incidence Supersonic similitude for Planar wedge, International Journal of Engineering research and Applications, Vol. 2, Issue 5, September-October 2012, pp. 468-471, ISSN: 2248-9622.

[13]. Asha Crasta, M. Baig, S.A. Khan, Estimation of Stability derivatives of a Delta wing in Hypersonic flow, International Journal of Emerging trends in Engineering and Developments in Vol.6, Issue2,Sep2012,pp505-516,ISSN:2249-6149.

[14]. Asha Crasta, S. A. Khan, Estimation of stability derivatives of an Oscillating Hypersonic delta wings with curved leading edges, International Journal of Mechanical Engineering \& Technology, vol. 3, Issue 3, Dec 2012, pp. $483-492$. ISSN 0976 - 6340 (Print), ISSN 0976 - 6359 (Online).

[15]. Asha Crasta and S. A. Khan, Stability Derivatives in the Newtonian Limit ,The International Journal of Advanced Research in Engineering and Technology,Volume:4,Issue:7, Nov-Dec 2013,pp .276-289.ISSN 0976 - 6480 (Print), ISSN 0976 - 6499 (Online).

[16]. S.A Khan, Asha crasta, 'Oscillating supersonic delta wings with curved leading edges', in international journal of Advanced studies in Contemporary Mathematics. Vol 20 ,No.3 July 2010, PP 359-372.

[17]. Asha Crasta and S. A. Khan, Hypersonic Similitude for Planar Wedges ,The International Journal of Advanced Research in Engineering and Technology,Volume:5,Issue:2, Feb 2014, pp .16-31.ISSN 0976 - 6480 (Print), ISSN 0976 - 6499 (Online).

[18]. Asha Crasta and Khan S. A., Stability derivatives of a delta wing with Straight leading edge in the Newtonian limit, International Journal of Engineering research and Applications, Vol. 4, Issue 3(version2), March 2014, pp. 32-38, ISSN: 2248-9622 